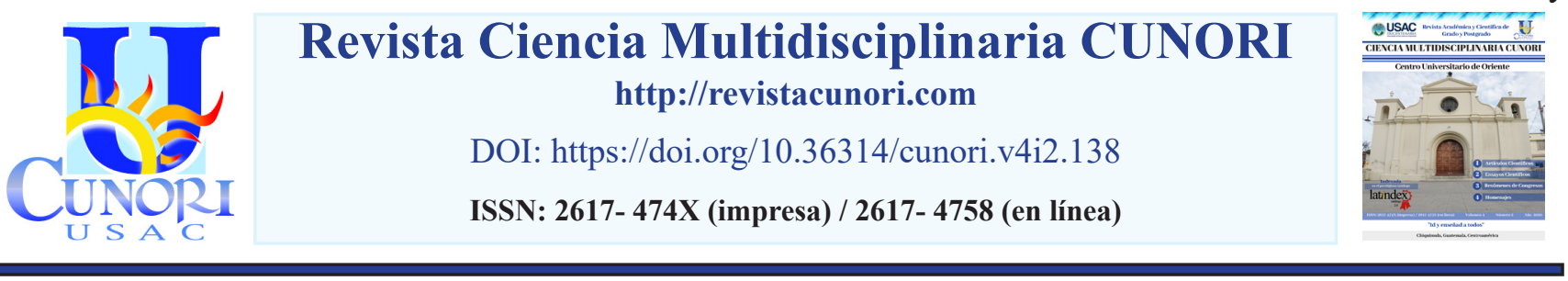

Referencia del ensayo

Orantes-Kestler, A. (2020). La Inteligencia Artificial y las oportunidades para la empresa en Guatemala. Revista Ciencia Multidisciplinaria Cunori, 4(2), 141-146. https://doi.org/10.36314/cunori.v4i2.138

\title{
La Inteligencia Artificial y las oportunidades para la empresa en Guatemala
}

\section{Artificial Intelligence and business opportunities in Guatemala}

\author{
Alejandro Orantes Kestler \\ Atlantic International University \\ https://orcid.org/0000-0003-3605-1419 \\ Recibido: 24 de marzo de 2020 / Revisado: 27 de marzo de 2020 / Aceptado: 28 de mayo de 2020 \\ Disponible en internet el 30 de octubre de 2020 \\ *Autor para correspondencia. \\ Correo electrónico: alejandroorantesk@gmail.com
}

Resumen

Guatemala ocupa el lugar 107 en el índice Global de Innovación publicado en el año 2019, los niveles de competitividad son

Jajos y por lo tanto la productividad es baja y en consecuencia las oportunidades a las que Guatemala puede acceder son limitadas y surge la importancia de evaluar si la Inteligencia Artificial puede ser una herramienta que ayude a las empresas a ser más productivas. Para realizar la investigación se recabó diferentes fuentes bibliográficas de actualidad, así como también se revisó lo que otros países como Estados Unidos han estado haciendo con la Inteligencia Artificial ya que tienen mayor ventaja en dichos temas, dentro de las referencias se identificó investigadores o profesores de MIT, Harvard o del Foro Económico Mundial ya que tienen dominio en lo relacionado a la Inteligencia Artificial. Como principal resultado se encontró que, aunque la Inteligencia Artificial aun no la utilizan mucho las empresas y sus usos aún están en una fase de exploración, quienes ya han comenzado son quienes tendrán mayor ventaja en el futuro.

Palabras clave: inteligencia artificial, innovación, estrategia, competitividad, productividad

Abstract

uatemala occupies the 107th place in the Glogal Innovation Index published in 2019, the levels of competitiveness are low and $\checkmark$ therefore productivity is low and consequently the opportunities that Guatemala can access are limited and the importance of evaluating if Artificial Intelligence can be a tool that helps companies to be more productive. To carry out the research, different current bibliographic sources were collected, as well as what other countries such as the United States have been doing with Artificial Intelligence as they have greater advantage in these topics, were reviewed, within the references researches or professors of MIT, Harvard or the World Economic Forum since they have dominance in relation to Artificial Intelligence. As a main result, it was found that although Artificial Intelligence is not yet used by too many companies and its uses are still in an exploration phase, those who have already started are those who will have the greatest advantage in the future.

Keywords: artificial intelligence, innovation, strategy, competitive, productivity 


\section{Introducción}

La innovación en la actualidad es una herramienta poderosa para darle una ventaja competitiva a las empresas, sin embargo Guatemala ocupa la posición 107 según el índice global de innovación y por otro lado existen herramientas como la Inteligencia Artificial que aún no es común ver su aplicación y esto hace que por no hacer uso de tecnologías innovadoras, las empresas no logren la productividad necesaria para ser competitiva, por tal motivo el presente ensayo busca identificar los beneficios y ventajas de implementar la Inteligencia Artificial, así como identificar las diferentes aplicaciones que tiene en las empresas y por último buscar que oportunidades existen para Guatemala, todo basado en identificar los principales autores o instituciones que han profundizado en el tema. Esta investigación es consecuencia de ensayos previos en los que el autor ha indagado sobre la relación entre la estrategia empresarial y la Inteligencia Artificial o también la relación de Big Data y los indicadores de desempeño, por lo que con la presente investigación la implicación es que el empresario guatemalteco descubra las áreas de oportunidad que existen y a nivel académico sirva para futuras investigaciones que permitan conocer a detalle las estrategias para aplicar de manera correcta la Inteligencia Artificial de tal manera que ayude a las empresas a ser más competitivas.

\section{Contenido}

Han pasado casi cuarenta años desde el estreno de la película Terminator y las personas veían todo como ciencia ficción y luego han salido películas como Her, Ex - Machina y actualmente series de televisión como Westworld en donde todas convergen en que la Inteligencia Artificial es una amenaza para la humanidad y todo debido a que la ambición de las personas ha provocado quitar ciertos controles a las computadoras y esto genera que con la Inteligencia Artificial la computadora tome el control y dada su capacidad de aprendizaje que se torna exponencial, resulta amenazando a los humanos hasta llevarlos a su destrucción. Sin embargo, estos escenarios apocalípticos lo único que reflejan de manera real es que en la actualidad la Inteligencia Artificial está teniendo una gran importancia y aun no reflejan la realidad de las posibilidades que existen detrás de la Inteligencia Artificial de manera real.

Comenzando por definir la Inteligencia Artificial y para ello la definición de inteligencia según RAE es la capacidad de comprender o de resolver problemas y la palabra artificial que significa que fue hecho o producido por el hombre y que es algo no natural, por lo que integrando ambas palabras se podría definir algo así como un producto hecho por el hombre que tiene la capacidad de comprender y resolver problemas. Sin embargo, la misma RAE define la Inteligencia Artificial como una disciplina informática para crear programas que se pueden comparar con la mente humana (Real Academia de la Lengua Española, 2020), por otro lado el sitio Salesforce.com define la IA como la creación de programas para que una computadora pueda replicar comportamientos humanos o en otras palabras que una computadora pueda pensar como un hombre (Salesforce, 2017).

Uno de los aspectos por lo que en la actualidad se hace tan importante la Inteligencia Artificial es que en la era de la información el Big Data se hace mas relevante ya que por medio de la programación se pueden definir funciones o instrucciones que provengan del Big Data como por ejemplo Facebook o Google Fotos que según las fotografías se sugieren agrupamientos o etiquetas de personas y esto dentro 
de una gama amplia de imágenes en toda la red y la complicación del Big Data radica en las cuatro principales características están identificadas por las cuatro V: Velocidad, Veracidad, Variedad y Volumen (Fundación Innovación Bankinter, 2019). Como referencia se tiene que de 33 zettabits en el año 2018 a un pronóstico de 175 zettabits en el año 2025 y Europa esta presupuestando 3.2 millardos de euros para IA mientras que Estados Unidos tiene un presupuesto de 12 millardos de USD y 6 millardos en Asia (European Commission, 2020).

Dentro de la Inteligencia Artificial se tienen diferentes áreas que son: Aprendizaje Automático que consiste en que la computadora realice funciones sin necesidad de una programación previa, y en este aspecto es donde más avances se están llevando a cabo. Luego esta el Aprendizaje Profundo se refiere a un aspecto mas complejo en donde se pueden relacionar imágenes por ejemplo que el sistema puede identificar una imagen en toda la red o también esta relacionado con el procesamiento de palabras como es el caso de un asistente como Alexa o Siri. Por último, esta el Análisis Predictivo que consiste en que por medio de recolectar una serie de datos o información relevante luego el algoritmo de Inteligencia Artificial puede predecir determinados resultados que ayudan a tomar mejores decisiones.

Por otra parte la Inteligencia Artificial lleva una alta relación con la ciencia de datos la cual se divide en cuatro grandes elementos que son: Analítica Descriptiva que solo describe datos históricos y el 90\% de las empresas la utiliza, analítica Diagnostica que intenta definir la causa de ciertos comportamientos, analítica Predictiva que se encarga de identificar probabilidades de aspectos que podrían suceder en el futuro y es utilizada por alrededor de un $13 \%$ de las empresas y por último la Analítica Prescriptiva la cual define o recomienda una acción a tomar basado en datos analizados (Santos, 2017).

Cabe resaltar que existe una diferencia importante entre la Ciencia de datos y la analítica de datos, ya que la ciencia de datos está enfocada en el descubrimiento de nuevos datos, investigación y uso de herramientas de programación, mientras que la analítica de datos busca el análisis de los datos ya definidos previamente y requiere un mayor dominio de la operación del negocio (Santos, 2017) y otros autores incluyen tambien al estratega de datos que esta en el medio de ambas disciplinas ya que su enfoque esta en detectar las necesidades de nuevos datos. Cabe recalcar que todo lo relacionado a la ciencia de datos y al Big Data esta totalmente relacionado con la inteligencia artificial, sin embargo no son sinonimos ya que como se definión anteriormente, la IA se refiere a la creación de algoritmos o programas que permiten crear comportamientos similares al del ser humano, mientras que los datos solamente son una materia prima para la IA.

Hablando de las ventajas y beneficios de utilizar la Inteligencia Artificial, el Machine Learning tiene la posibilidad de brindar ventajas como la de tener información precisa sobre las tendencias y la evolución de precios del mercado, otras herramientas como Watson de IBM permiten monitorear en tiempo real las tendencias del mercado, generar una interpretación y apoyar a la empresa para tomar mejores decisiones. También menciona la comisión europea (2020) que para los ciudadanos también existen beneficios como en el sector de salud, transportes mas limpios, reducción de paros de maquinaria, en general mejores servicios públicos. Para el desarrollo de negocios se podrán crear nuevos negocios y 
servicios en áreas en donde se tengan fortalezas y para los intereses públicos la reducción de costos en los servicios como transporte, energía eléctrica, salud, educación.

Dentro de las aplicaciones más comunes están los buscadores de productos que se enfocan en sugerir productos según el perfil del cliente, chatbots que sirve para tener una comunicación con los clientes, recomendadores que sirven para hacer ventas cruzadas al cliente, tal como lo maneja Amazon o Netflix, prevención del fraude que según ciertos comportamientos la IA puede generar alertas de posibles fraudes en las empresas o instituciones (Basco, 2016).

Según una investigación de la empresa de tecnología Accenture, el 67\% de los trabajadores considera importante capacitarse en Inteligencia Artificial (MIT SMR Connections, 2020). Dentro de los beneficios Sejnowski (2018) refiere que existe una tendencia de que para el 2025 la IA solo en el ámbito de reconocimiento y clasificación de imágenes podría generar ocho millardos de dólares, por otro lado en un estudio del MIT Bughin (2018) indica que las empresas que utilicen Inteligencia Artificial podrían aumentar sus ventas hasta por 10\%, la gran oportunidad es que el 95\% de las empresas no han adoptado la tecnología y la cifra alarmante es que según las simulaciones realizadas por McKinsey establecen que quienes adopten la IA podrán duplicar sus ventas para el 2030.

\section{Aplicación de la Inteligencia Artificial}

Por ejemplo, la herramienta de Inteligencia Artificial de la empresa Salesforce llamada Einstein que, por medio del CRM al momento de prospectar clientes, el algoritmo puede indicar cuales son los clientes que realmente requieren un seguimiento para lograr la venta y cuales deben ser descartados, con ello se logra mayor efectividad de las ventas (Salesforce, 2017). También esta Corea del Sur con diferentes empresas que están integrando a su proceso de reclutamiento y entrevistas la Inteligencia Artificial y esta herramienta es capaz de organizar una entrevista y por medio de reconocimiento facial e identificación de sus gestos, se puede detectar si el candidato satisface los requerimientos de la posición (Cha, 2019).

Aplicaciones en Siri de Apple, Asistente de Google de la empresa Google, Alexa de Amazon, entre otros, los cuales se utilizan con un procesamiento de lenguaje natural y permite resolver problemas como pedirle información publicada en la web, habilitar habilidades personalizadas como dar las noticias o aprender inglés por medio de prácticas diarias.

En cuanto a aplicaciones como la de Amazon que brinda sugerencias para compra de productos basado en las compras anteriores o caso similar el caso de Netflix que también sugiere películas basado en las anteriores películas vistas. De manera similar el caso de la aplicación Waze que brinda información sobre el trafico mediante la integración del GPS y el volumen de usuarios que viajan por la misma ruta se puede establecer las mejores rutas para optimizar el tiempo de llegada a un lugar. 
Las oportunidades para Guatemala son muy amplias ya que como país no hay nada estructurado y caso contrario países como Estados Unidos o regiones como Europa o Asia que tienen un presupuesto y una política en la que definen el marco de trabajo para integrar el ámbito empresarial, gobierno, academia, entre otros, en Guatemala aún hay un largo camino por recorrer, y eso por ello que haciendo un consolidado de las diferentes propuestas de como implementarlo a nivel país se pueden mencionar los siguientes pasos:

A nivel de empresas: capacitarse, identificar individuos con potencial para los roles relacionados con Inteligencia Artificial, comenzar a construir datos, explorar nuevas habilidades requeridas en IA y mantener este circulo virtuoso para ir perfeccionando las capacidades (MIT SMR Connections, 2020).

A nivel general como gobierno: Hacer un plan de acción, establecer una organización que a nivel nacional tenga presupuesto y a su vez la responsabilidad de desarrollar las aplicaciones de IA, crear centros de desarrollo de IA, crear y ejecutar un plan de capacitación en desarrollo de habilidades de IA en la fuerza laboral, alianzas o sociedades entre el gobierno y el sector privado.

\section{Conclusión}

La Inteligencia Artificial es una herramienta que brinda grandes beneficios y ventajas a las empresas y así se ha evidenciado en gran variedad de empresas en diferentes países del mundo, sin embargo, en Guatemala aun no se explota la IA y existen grandes oportunidades que permitirían que las empresas logren mayores ventajas. Como primeros pasos a nivel individual las empresas deben llevar a cabo un diagnostico que les permita en que áreas pueden implementar la Inteligencia Artificial y adicional identificar la capacitación necesaria para que posteriormente puedan aplicarlo dentro de las empresas. A nivel de gobierno las oportunidades son amplias, pero se debe brindar mayor presupuesto al Senacyt y al Concyt para que puedan generar mas incentivos para el desarrollo de la Inteligencia Artificial para que sea parte de una política de país y de esta manera se logre aumentar las ventajas competitivas de Guatemala.

\section{Referencias}

Basco, P. (2016). Machine learning', una gran ventaja competitiva digital. ThinkBig/Empresas.

Bughin, J. (2018). Wait and see could be a costly AI Strategy. Foro Economico Mundial.

Cha, S. (2019). In South Korea, you can take a class on dealing with an AI hiring bot. Foro Economico Mundial.

European Commission. (2020). On Artificial Intelligence - A European approach to excellence and trust. Bruselas: European Commission.

Fundación Innovación Bankinter. (2019). Big Data: El poder de los datos. Fundación Innovación Bankinter.

MIT SMR Connections. (2020). Transform Your Workforce With Skills for Machine Learning. Massachusetts Institute of Technology. 
Real Academia de la Lengua Española. (2020). RAE. Obtenido de RAE: https:/dle.rae.es/ inteligencia\#2DxmhCT

Salesforce. (2017). salesforce.com. Obtenido de salesforce.com: https://www.salesforce.com/mx/ blog/2017/6/Que-es-la-inteligencia-artificial.html

Santos, P. R. (2017). Analiticas Big Data, si, ¿pero cuáles? Think Big.

Santos, P. R. (2017). Data Science vs Data Analytics: parecidos, pero no iguales. Obtenido de https://empresas.blogthinkbig.com/data-science-vs-data-analitycs/

Sejnowski, T. (2018). AI will make you smarter. Here’s how. Foro Economico Mundial.

\section{Sobre el autor}

\section{Alejandro Orantes Kestler}

Docente universitario a nivel de maestrías por más de ocho años en diferentes universidades. Tiene una licenciatura en Administración de Empresas, Maestría en Negocios Internacionales, una segunda Maestría en Dirección de Marketing, graduado de Doctorate of Business Administration en Atlantic International University y adicional pensum cerrado de doctorado de Ciencias Empresariales y candidato a privado de tesis con su investigación sobre la innovación estratégica en la empresa guatemalteca, la que será su cuarta tesis a publicar.

Copyright (c) Alejandro Orantes Kestler

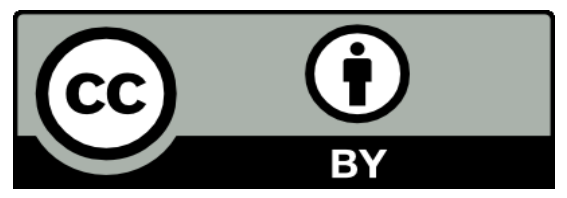

Este texto está protegido por una licencia CreativeCommons 4.0.

Usted es libre para compartir, copiar y redistribuir el material en cualquier medio o formato y adaptar el documento, remezclar, transformar y crear a partir del material para cualquier propósito, incluso comercialmente, siempre que cumpla la condición de atribución: usted debe reconocer el crédito de una obra de manera adecuada, proporcionar un enlace a la licencia, e indicar si se han realizado cambios. Puede hacerlo en cualquier forma razonable, pero no de forma tal que sugiera que tiene el apoyo del licenciante o lo recibe por el uso que hace. 